\title{
TUG Test Instrumentation for Parkinson's disease patients using Inertial Sensors and Dynamic Time Warping
}

\author{
M. Reyes Adame ${ }^{1}$, A. Al-Jawad ${ }^{2}$, M. Romanovas ${ }^{2}$, M.A. Hobert ${ }^{4,5}$, W. Maetzler ${ }^{4,5}$, K. Möller ${ }^{1}$, Y. Manoli ${ }^{2,3}$ \\ ${ }^{1}$ Department of Biomedical Engineering, Hochschule Furtwangen University, Villingen-Schwenningen, Germany, \\ E-Mail: miguel.reyes.adame@gmail.com \\ ${ }^{2}$ Institut für Mikro- und Informationstechnik der Hahn-Schickard Gesellschaft e.V. (HSG-IMIT), Villingen- \\ Schwenningen, Germany \\ ${ }^{3}$ Fritz Huettinger Chair of Microelectronics, Department of Microsystems Engineering - IMTEK, University of \\ Freiburg, Germany \\ ${ }^{4}$ Hertie Institute for Clinical Brain Research, Department of Neurodegeneration, Center of Neurology, University of \\ Tuebingen, Tuebingen, Germany \\ ${ }^{5}$ DZNE, German Center for Neurodegenerative Diseases, Tuebingen
}

\begin{abstract}
The Timed Up and Go (TUG) test is a clinical tool widely used to evaluate balance and mobility, e.g. in Parkinson's disease (PD). This test includes a sequence of functional activities, namely: sit-to-stand, 3-meters walk, $180^{\circ}$ turning, walk back, and turn-to-sit. The work introduces a new method to instrument the TUG test using a wearable inertial sensor unit (DynaPort Hybrid, McRoberts B.V., NL) attached on the lower back of the person. It builds on Dynamic Time Warping (DTW) for detection and duration assessment of associated state transitions. An automatic assessment to substitute a manual evaluation with visual observation and a stopwatch is aimed at to gain objective information about the patients. The algorithm was tested on data of 10 healthy individuals and 20 patients with Parkinson's disease (10 patients for early and late disease phases respectively). The algorithm successfully extracted the time information of the sit-to-stand, turn and turn-to-sit transitions.
\end{abstract}

\section{Introduction}

Actually about five million people suffer from Parkinson's disease (PD) worldwide, making it the second-most common neurodegenerative disease after Alzheimer's disease [1,2]. Typical symptoms of PD are movement deficits such as tremor, rigidity, bradykinesia, and postural instability. These symptoms are mainly caused by the death of dopaminergic neurons in the midbrain [3].

It is important to monitor the progression of PD to provide physicians and patients with timely feedback regarding the state and progress of the disease. Furthermore the effectiveness of therapeutic measures e.g. medication dosage can be better monitored and optimized. Observation of motor symptoms by the doctor and evaluation by use of diaries are subjective and not sensitive to subtle changes while the monitoring via camera systems is also expensive and time-consuming [4]. Assessment with small ambulatory inertial sensors including e.g. wireless transmission is unobtrusive and thus an attractive alternative to common observation methods [5].

The main goal of the presented work is to evaluate the application of inertial sensors and corresponding algorithms to automate the Timed Up and Go (TUG) test for differentiation between healthy individuals and patients with PD. The TUG test is one typical and popular disease assessment methodology [6] used to evaluate balance ability, fall risk, and mobility of a person with PD. During the test the person is asked to stand up from a chair and walk 3-meters distance at convenient speed, followed by a turn of $180^{\circ}$, walk back, and sit on the chair after another turn of $180^{\circ}$ (see fig. 1). The timing information of each

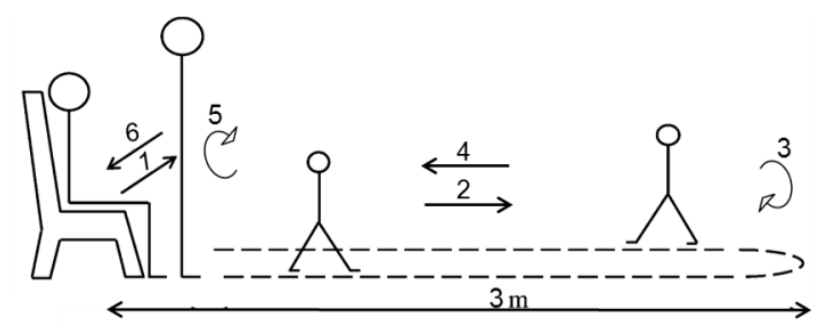

Fig. 1: Schematic representation of different transitions (1-6) during the Timed Up and Go test. (1) sit-to-stand, (2) walking, (3) first turning, (4) walking back, (5) second turning and (6) stand-to-sit. Modified from [8].

of the subphases taken from the test is clinically important to assess the rehabilitation process and the treatment for patients [4]. Using a stopwatch to measure this time information is difficult and consumes clinical resources when applied to a larger number of patients. Moreover, the accuracy especially in combination with a limited clinicians' experience is arguable. The result might not have the precision needed to objectively assess the effect of rehabilitative intervention [4]. Hence, an inertial sensor unit is addressed in this work to improve the process of evaluation and to get more accurate estimates.

Recently several approaches have been proposed to automate the TUG test based on inertial sensors [4, 7-10]. For example, Salarian et al. [4] detected and separated transition components of the TUG test using accelerometer and gyroscope sensors which were attached to the limbs as well as to the trunk. By using a trained statistical classifier he could show a significant difference between early PD patients and a control group, in contrast to the results obtained with a stopwatch and visual observations. 
Yet another group employed a hidden Markov model (HMM) to recognize the different time phases of the TUG test [7]. The authors combined the HMM with different classifiers and compared their performances by analyzing the different phases of the TUG test.

In this work a method based on Dynamic Time Warping (DTW) is applied for time alignment of transition phases using a single 3-axis gyroscope mounted on the lower back of the person. Similar techniques were recently employed for scenarios of a general activity classification $[11,12]$.

\section{Materials and Methods}

Ten PD patients (age range: 41-69 and average: $58.8 \pm 9.5$, gender: 2 female and 8 male) at an early stage of the disease (Hoehn and Yahr score between 1 and 2), ten patients (age range: 60-77 and average: 66.2 \pm 4.8 , gender: 3 female and 7 male) in a slightly advanced stage (Hoehn and Yahr score between 2 and 3), and ten healthy individuals (age range: 51-76 and average: 63.2 \pm 10.1 , gender: 6 female and 4 male) were recruited from the university hospital of Tuebingen for this study to perform the TUG test. The participants were asked to execute the test twice. The measurements were recorded with an inertial sensor unit placed on the lower back. The study was approved by the local ethical committee, and all participants gave written informed consent.

The participants wore an inertial sensor unit (DynaPort Hybrid, McRoberts B.V., NL) [13]. This unit includes a 3-axis accelerometer (range: $\pm 2 \mathrm{~g}$, resolution: $1 \mathrm{mg}$ ) and a 3 -axis gyroscope (range: $\pm 100 \mathrm{deg} / \mathrm{s}$, resolution: $0.0069 \mathrm{deg} / \mathrm{s}$ ) with a sampling rate of $100 \mathrm{~Hz}$. The data were stored on a Micro-SD card for further processing. Within the presented work we used only the gyroscope signals. The algorithms were implemented and tested in MATLAB (version 7.10 (R2010a)). Before the analysis step were carried out, the signals were low-pass filtered using a non-causal moving average filter with a length of 31 .

Here we focus on the detection of the sit-to-stand, turning, and turn-to-sit transitions. Sit-to-stand transition was analyzed and detected with the signals from the gyroscope along the pitch axis, while the yaw angular rate signal was used for the turn transition. For the detection of the turn-to-sit transition both signals were evaluated. The start of the turn transition was detected by analyzing the yaw angular rate signal, while the end was recognized with the pitch axis signal. The reference data were extracted manually from the raw sensor signals by defining start and end points of every transition.

Since the TUG test is performed by a series of known motion types in a sequential way, an algorithm which aligns signals with different lengths and contains time information can be applied. Here we propose to employ DTW for the time alignment between the measurements and a motion pattern with known phase time correspondence. The DTW algorithm is chosen due to its wellknown efficiency and robustness in performing temporal fusion [15]. This algorithm finds an optimal alignment between two sequences $X=\left\{x_{1}, x_{2}, \ldots, x_{n}\right\}$ of length $n$ and $Y=\left\{y_{1}, y_{2}, \ldots, y_{m}\right\}$ of length $m$. The test sequence is normalized to the template by upsetting and stretching the

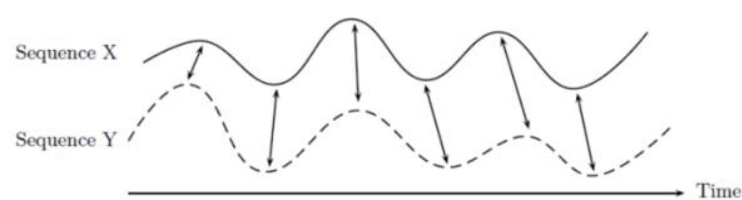

Fig. 2: Alignment of two time sequences. The Arrows mark aligned points of the two sequences. Taken from [14].

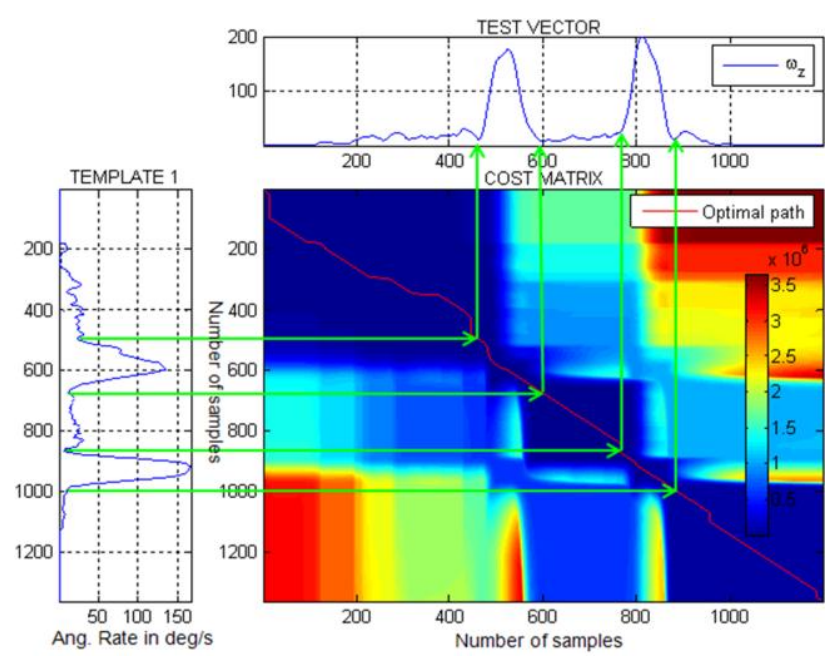

(a) Alignment of angular rate signals in yaw axis

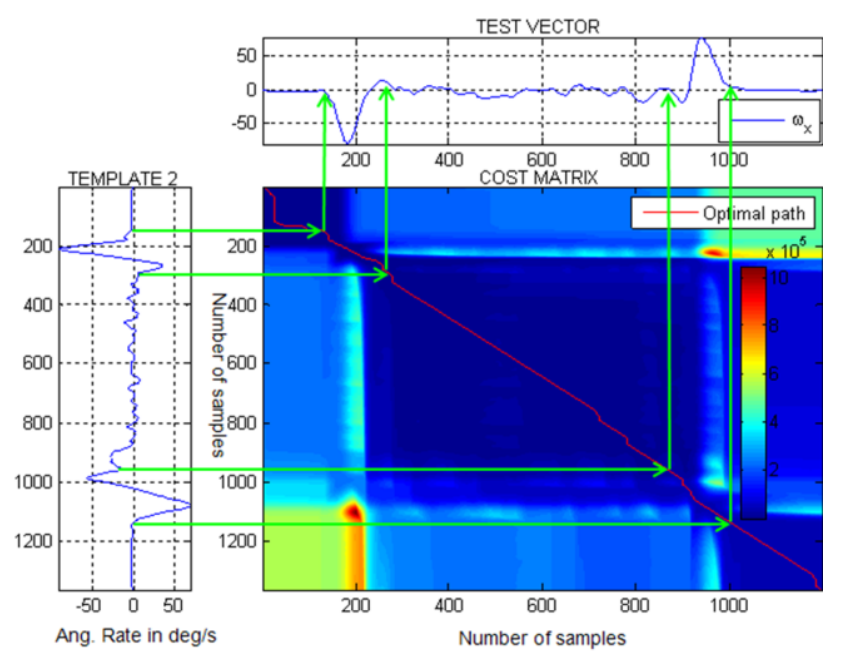

(b) Alignment of angular rate signals in pitch axis

Fig. 3: Optimal warping path between a template (left) and a pattern (top) of (a) ang. rate signals in the yaw-axis and (b) ang.rate signals in the pitch-axis. Ang. Rate in deg/s (y-axis) over signal length in number of samples ( $\mathrm{x}$-axis).

signal segments so that similar sensor observations (see fig. 2) occur as far as possible at the same place in both sequences. The algorithm finds the optimal alignment by building an $n$-by- $m$ cost matrix where each element $(i, j)$ corresponds to the square of the pairwise Euclidean distance:

$$
d(i, j)=\left(x_{i}-y_{j}\right)^{2}
$$

between the two points in the $\mathrm{X}$ and $\mathrm{Y}$ sequences [16]. Each set of the matrix elements starting at upper left corner and ending in lower right corner, defines an alignment between $\mathrm{X}$ and $\mathrm{Y}$ sequence called a warping path. The optimal warping path $W_{D T W}$ is the path where the total sum of the local distances along the path $d\left(w_{k}\right)$ is minimal:

$$
W_{D T W}(X, Y)=\arg \min _{w} \sum_{k=1}^{K} d\left(w_{k}\right)
$$


This optimal alignment path can be calculated using dynamic programming techniques [16]. The phase time information is mapped by the backtracking through the obtained optimal path. Fig. 3 shows the optimal paths (red lines) through the n-by-m cost matrix for the angular rate signals in yaw (a) and pitch (b) axis for one of our measurements.

\section{Results}

The optimal path through the cost matrix was calculated for all 60 measurements. The cost matrices and the paths for a representative assessment are shown in fig. 3 . For an accurate evaluation of the algorithm's performance, the output of the DTW was compared to reference data (table 1). The control group completed the TUG test slightly faster than the early PD group: $9.88 \mathrm{~s} \pm 1.78 \mathrm{~s}$ vs. $10.67 \mathrm{~s} \pm 1.9 \mathrm{~s}$ (reference measurements), $10.05 \mathrm{~s} \pm 1.75$ $\mathrm{s}$ vs. $10.76 \mathrm{~s} \pm 1.76 \mathrm{~s}$ (DTW algorithm). The advanced PD group needed more time for performing the TUG test, than both other cohorts: $12.19 \mathrm{~s} \pm 2.67 \mathrm{~s}$ (reference measurements) and $12.03 \mathrm{~s} \pm 2.61 \mathrm{~s}$ evaluated with the algorithm.

The state transitions sit-to-stand, turning and turn-tosit were all detected automatically by the algorithm. The results for the mean duration of the transitions are summarized in table 2. Clearly, the accuracy of the DTW strongly depends on the template selection. Here, for each group a different template was chosen by an explicit search process. With a larger measurement set available, one of the automatic template selection methods will be adopted or automatic reference template design methods will be employed [15].

Table 1 shows the mean and maximum absolute errors when detecting start and end times of the transitions. The results for the sit-to-stand transitions were not estimated because it was difficult to define a reference on the raw data for the start time of the stand-to-sit transition. Therefore, the start of the second turn and the end of the standto-sit transition were detected so that both transitions could be combined into a single turn-to-sit transition.

The walking phase could be evaluated by measuring the period from the end of the sit-to-stand phase to the be- ginning of the (first) turn, and from the end of the turning to the start of the second turning. This indirect evaluation is not very representative as it is not possible to distinguish between walking and probable standing phases before or after the walking phase.

The significant difference between the groups was checked using the Wilcoxon rank sum test. The p-values are shown in table 3 .

\section{Discussion}

The main attention was set to present a novel technique for obtaining the time duration of state transitions. The results in table 2 confirm that the algorithm achieves a reasonably good performance when compared to the provided reference data. Differences detected between controls and early PD patients, and between these two groups and advanced PD patients further argue for the ecological validity of the algorithm which, however, needs certainly further evaluation.

Only the deviation between the algorithm output and the reference for the sit-to-stand transition among the healthy control group indicates that the algorithm needs further improvement. Although the algorithm showed small mean errors in detecting the start and end points of the state transitions, some outliers can still be found (see entries for max errors in table 1.).

However, mentioned differences might at least partly be explained by a non-negligible error being present in the reference data due to manual extraction of the reference timing from raw signals without direct visual feedback for the presented measurement set, and not by a bias of the algorithm per se.

In contrast to Salarian et al. [4], our results for the duration of the transitions, of both the reference and algorithm output, show no significant difference between the healthy control and early PD group (table 3 ). The comparison of the group with the patients in an advanced state of the disease (LTPD) with the control or the early PD (STPD) group confirmed significant differences in the sitto-stand and turning transitions. This confirms the principle relevance for clinical use of this algorithm. The sit-tostand duration from the reference data for the control vs.

Table 1: Algorithm absolute error deviations with respect to reference by detecting the start and end points of the state transitions.

\begin{tabular}{l|cccccc}
\hline & \multicolumn{2}{|c}{ Control Group } & \multicolumn{2}{c}{ Early PD Group } & \multicolumn{2}{c}{ Long Time PD Group } \\
\hline Activity & Mean (s) & Max (s.) & Mean (s) & Max (s) & Mean (s) & Max (s) \\
Start Sit-to-Stand & 0.06 & 0.22 & 0.06 & 0.19 & 0.32 & 0.52 \\
End Sit-to-Stand & 0.25 & 0.90 & 0.19 & 0.81 & 0.21 & 0.74 \\
Start Turn & 0.34 & 1.43 & 0.31 & 0.94 & 0.28 & 0.71 \\
End Turn & 0.15 & 0.49 & 0.17 & 0.41 & 0.14 & 0.44 \\
Start Turn-to-Sit & 0.14 & 0.70 & 0.14 & 0.49 & 0.27 & 0.85 \\
End Turn-to-Sit & 0.40 & 0.95 & 0.38 & 0.81 & 0.27 & 0.73 \\
\hline
\end{tabular}

Table 2: Time duration for the state transitions of the TUG-Test. S.D. - Standard Deviation

\begin{tabular}{|c|c|c|c|c|c|c|c|c|c|c|c|c|}
\hline \multirow[b]{3}{*}{ Activity } & \multicolumn{4}{|c|}{ Control Group } & \multicolumn{4}{|c|}{ Early PD Group } & \multicolumn{4}{|c|}{ Long Time PD Group } \\
\hline & \multicolumn{2}{|c|}{ Reference (s) } & \multicolumn{2}{|c|}{ Algorithm (s) } & \multicolumn{2}{|c|}{ Reference (s) } & \multicolumn{2}{|c|}{ Algorithm (s) } & \multicolumn{2}{|c|}{ Reference (s) } & \multicolumn{2}{|c|}{ Algorithm (s) } \\
\hline & Mean & S.D. & Mean & S.D. & Mean & S.D. & Mean & S.D. & Mean & S.D. & Mean & S.D. \\
\hline Sit-to-Stand & 1.62 & 0.42 & 1.34 & 0.40 & 1.45 & 0.30 & 1.27 & 0.21 & 1.87 & 0.44 & 1.81 & 0.39 \\
\hline First Turn & 1.91 & 0.36 & 1.97 & 0.55 & 2.07 & 0.31 & 1.89 & 0.35 & 2.54 & 0.57 & 2.69 & 0.62 \\
\hline Turn-to-Sit & 3.63 & 0.85 & 3.96 & 0.95 & 3.80 & 0.99 & 3.83 & 0.85 & 4.20 & 1.03 & 4.12 & 1.10 \\
\hline
\end{tabular}


Table 3: Comparison of the state transition durations between the control, long time PD (LTPD) and the short time PD (STPD) group for the algorithm (alg.) and reference (ref.) outputs. The values marked in bold were significantly different $(\mathrm{p}<0.05)$.

\begin{tabular}{lccc}
\hline \multicolumn{3}{c}{ Rank sum p-values } \\
\hline & Control vs. LTPD & Control vs. STPD & STPD vs. LTPD \\
\hline Sit-to-stand (alg.) & $\mathbf{0 . 0 0 1 2}$ & 0.8392 & $\mathbf{0 . 0 0 0 0 1 5}$ \\
Sit-to-stand (ref.) & 0.0601 & 0.2974 & $\mathbf{0 . 0 0 1 5}$ \\
\hline Turning (alg.) & $\mathbf{0 . 0 0 0 3}$ & 0.9784 & $\mathbf{0 . 0 0 0 0 2 6}$ \\
Turning (ref.) & $\mathbf{0 . 0 0 0 4}$ & 0.1297 & $\mathbf{0 . 0 0 3 3}$ \\
\hline Turn-to-sit (alg.) & 0.5608 & 0.5883 & 0.4093 \\
Turn-to-sit (ref.) & 0.0834 & 0.4818 & 0.2035 \\
\hline
\end{tabular}

the LTPD group showed in contrast to the algorithm output no significant difference. Again we may speculate that the manual referencing of the transitions from the raw signals was not accurate enough.

The turn-to-sit transition showed also no significant difference between all groups. This indicates that combined timing information can be not accurate enough for reliable group separation. Analysis of the single transitions will be considered within future work.

A temporal signal fusion can also be achieved by alternative mathematical frameworks such as HMMs. However, according to [15], the DTW has some advantages compared to alternative frameworks. It is numerically more efficient, accurate and requires very few tuning. Moreover, differently from HMM-based methods, the DTW shows higher robustness and can be designed avoiding explicit training phases. Note that the latter often requires a significant number of labeled experimental data which may be simply not available. Similarly to HMM, the DTW approach can be easily extended for advanced multidimensional features and augmented with feature dimensionality reduction methods. A clear disadvantage of DTW is that the framework is not suitable for arbitrary (or unknown) sequence of known motion patterns and is, in general, less powerful when compared to advanced versions of HMMs.

\section{$5 \quad$ Conclusions and Future Work}

If an exact chronology of the state sequences is known, the DTW can be efficiently applied for time alignment between pattern and test measurement. The method can be seen as a promising alternative to monitor the progress of PD when applied for the TUG test.

Our next steps will focus on improving the accuracy of temporal estimation of the start and end points of the state transitions. Some performance improvement is also expected when employing a multiple template approach or an implicit template construction as suggested in [15]. We are also working on an automated template selection criterion to find the best template, which can be used for all groups. Moreover, the validation of the algorithm performance should be augmented with video for reference timing extraction.

\section{References}

[1] Chen JJ "Parkinson's disease: health-related quality of life, economic cost, and implications of early treatment." Am J Manag Care. 2010 Mar;16 Suppl Implications:S87-93. Review.
[2] Simo S. Oja, Arne Schousboe, Pirjo Saransaari, Abel Lajtha "Handbook of Neurochemistry and Molecular Neurobiology: Amino Acids and Pep-tides in the Nervous System" 3rd edition, Springer Verlag, 2007, ISBN-10: 0387303421

[3] Maetzler W, Liepelt I, Berg D. Progression of Parkinson's disease in the clinical phase: potential markers. Lancet Neurol 2009; 8: 1158-71.

[4] Salarian A, Horak FB, Zampieri C, Carlson-Kuhta P, Nutt JG, Aminian K. iTUG, a sensitive and reliable measure of mobility. IEEE Trans Neural Syst Rehabil Eng. 2010 Jun;18(3):303-10. Epub 2010 Apr 12.

[5] Grimaldi G, Manto M. Neurological tremor: sensors, signal processing and emerging applications. Sensors (Basel). 2010;10(2):1399-422.

[6] Brusse KJ, Zimdars S, Zalewski KR, et al. Testing functional performance in people with Parkinson disease. Phys Ther. 2005; 85:134-141.

[7] Pierre Jallon, Benjamin Dupre and Michel Antonakios "A graph based method for timed up and go test qualification using inertial sensors", 2011 IEEE International Conference on Acoustics, Speech and Signal Processing (ICASSP), May 2011, Pages: 689-692

[8] Luca Palmerini, Sabato Mellone, Laura Rocchi, Lorenzo Chiari "Dimensionality reduction for the quantitative evaluation of a smartphone-based Timed Up and Go test", IEEE Engineering in Medicine and Biology Society, Aug 2011

[9] Weiss A, Herman T, Plotnik M, Brozgol M, Giladi N, Hausdorff JM. "An instrumented Timed Up and Go: the added value of an accelerometer for identifying fall risk in idiopathic fallers". Physiol Meas. 2011 Dec;32(12):2003-18.

[10]Higashi, Y.; Yamakoshi, K.; Fujimoto, T.; Sekine, M.; Tamura, T.; , "Quantitative evaluation of movement using the timed up-and-go test," Engineering in Medicine and Biology Magazine, IEEE, vol.27, no.4, pp.38-46, July-Aug. 2008

[11]Sasiwan Paiyarom, Poj Tungamchit, Rachapor Keinprasit, Prakasith Kayasith "Activity Monitoring System using Dynamic Time Warping for the Elderly and Disabled people", Computer, Control and Communication, 2009, IEEE 2009

[12]Rossana Muscillo, Silvia Conforto, Maurizio Schmid, Paolo Caselli, Tommaso D' Alessio "Classification of motor activities through derivative dynamic time warping applied on accelerometer data", Engineering in Medicine and Biology Society, 2007. EMBS 2007

[13]McRobersts Moving Technology. [Online]. Available: http://www.mcroberts.nl [last accessed: march 2012]

[14]Müller M., "Information Retrieval for Music and Motion", Springer-Verlag 2007, Chapter 4 Dynamic Time Warping

[15]Ming Hsiao Ko, Geoff West, Svetha Venkatesh, Mohan Kumar, "Using dynamic time warping for online temporal fusion in multisensor systems", Information Fusion, Volume 9, Issue 3, July 2008, Pages 370-388, ISSN 1566-2535

[16]H. B. Mitchell, "Multi-Sensor Data Fusion: An Introduction”, 1st ed. Springer, Sep. 2007. 